\title{
The fog, the attractive and the addictive: pulmonary effects of vaping with a focus on the contribution of each major vaping liquid constituent
}

\author{
Ariane Lechasseur ${ }^{1,2}$ and Mathieu C. Morissette ${ }^{1,3}$ \\ Affiliations: ${ }^{1}$ Quebec Heart and Lung Institute, Université Laval, Quebec, Canada. ${ }^{2}$ Faculty of Medicine, \\ Université Laval, Quebec, Canada. ${ }^{3}$ Dept of Medicine, Université Laval, Quebec, Canada. \\ Correspondence: Mathieu C Morissette, Quebec Heart and Lung Institute, Dept of Medicine, Université Laval, \\ 2725 Chemin Sainte-Foy, Quebec, Canada, G1V 4G5. E-mail: mathieu.morissettedcriucpq.ulaval.ca
}

@ERSpublications

A review of what is currently known of the pulmonary effects of vaping with special emphasis on the specific effects of each e-liquid constituents https://bit.ly/2GlfTji

Cite this article as: Lechasseur A, Morissette MC. The fog, the attractive and the addictive: pulmonary effects of vaping with a focus on the contribution of each major vaping liquid constituent. Eur Respir Rev 2020; 29: 200268 [https://doi.org/10.1183/16000617.0268-2020].

ABSTRACT Vaping has become increasingly popular over the past decade. This pragmatic review presents the published biological effects of electronic cigarette vapour inhalation with a focus on the pulmonary effects. Special attention has been devoted to providing the documented effects specific to each major ingredient, namely propylene glycol/glycerol, nicotine and flavouring agents. For each ingredient, findings are divided according to the methodology used, being in vitro studies, animal studies and clinical studies. Finally, we provide thoughts and insights on the current state of understanding of the pulmonary effects of vaping, as well as novel research avenues and methodologies.

\section{Introduction}

The use of electronic (e)-cigarettes or devices, also known as vaping, has become increasingly prevalent over the past 10-12 years [1]. Initially marketed as a cessation tool for tobacco smoking, e-cigarettes are now being used by never-smokers, being highly popular among adolescents and young adults [2]. E-cigarette use has surpassed combustible tobacco cigarette use in adolescents in the USA [3]. The disconnect between the number of e-cigarette users and the scientific knowledge on its pulmonary effects is astonishing. While unravelling the complete nature of the pulmonary effects of vaping represents a daunting task, research groups around the world have tackled this scientific problem and continue to uncover the short- and long-term health effects of vaping. In this review, we present specific effects of each major vaping liquid ingredient, as well as the undistinguishable effects. We also share thoughts on conceptual and methodological avenues that are/could be taken by scientists to pursue basic and translational research on the pulmonary effects of vaping.

\section{Effects specific to each vaping liquid constituent}

E-cigarettes allow nicotine and flavouring agents to be delivered into the lungs through a heat-mediated vaporisation process. When activated, a battery-powered heating coil rapidly heats a liquid, often called e-liquid or e-juice, leading to its atomisation and easy inhalation. The main constituents of e-liquids are

Provenance: Commissioned article, peer reviewed

Received: 12 Aug 2020 | Accepted after revision: 25 Sept 2020

Copyright $\odot$ ERS 2020. This article is open access and distributed under the terms of the Creative Commons Attribution Non-Commercial Licence 4.0. 
propylene glycol and glycerol, which act as vehicles for various concentrations of nicotine and different types of flavouring agents. During their shelf live and through the atomisation process, e-liquid constituents are likely to interact, oxidise and generate potentially harmful by-products [4]. Some of these chemicals have been identified, such as free radicals, heavy metals, acrolein, carbonyls, formaldehyde and acetaldehyde [5-7]. However, the sheer number of commercial e-liquids makes it hard to identify and study the entire chemical compounds present in e-cigarette vapours, and the majority of them remain unknown. Thus in this review we will address the physiological impact of e-cigarette based on the formulation of vaping liquids, not vapours, with the main chemicals being propylene glycol, glycerol, nicotine and flavour molecules.

\section{The fog: propylene glycol and glycerol}

Propylene glycol (propane-1,2-diol) and glycerol (vegetable glycerine) are the common denominators of e-liquids. Propylene glycol and glycerol allow for the solubilisation of nicotine and flavours into a homogenous solution and are also responsible for the fog-like nature of e-cigarette vapours. Both compounds have long being used by the food industry as sweeteners or stabilising agents [8]. While they are considered safe to ingest [9], the consequences of propylene glycol and glycerol inhalation remain unknown despite worldwide availability of e-cigarettes. Earlier reports indicated increased upper airway symptoms and lower lung functions for entertainment workers chronically exposed to theatrical fogs, composed of propylene glycol and other glycols [10]. The next sections will focus on the impact of propylene glycol and glycerol exposure alone, without nicotine or flavourings.

\section{In vitro studies}

\section{Cell viability and gene expression profile}

Several studies reported no changes in cell viability when exposing lung epithelial cells [11-13], lung fibroblasts [14], embryonic stem cells [14] or monocytes [15] to propylene glycol and/or glycerol. Other studies reported increased cell death following exposure to propylene glycol vapours [16, 17], glycerol vapours [17] or both [18, 19]. It also triggers an oxidative stress response, with elevated expression of antioxidant enzymes glutathione peroxidase 2 (GPX2), NAD(P)H quinone dehydrogenase 1 (NQO1) and heme oxygenase-1 (HO-1) [17, 20]. Glycerol vapours alone increased mRNA levels of HO-1, cellular stress-related genes, as well as carbonylated protein levels, suggesting increased cellular stress [16]. Genome-wide expression arrays conducted in primary human bronchial epithelial cells exposed to propylene glycol and glycerol for $24 \mathrm{~h}$ indicated minimal changes overall, with variations in ribosomal protein S8 (RPS8), zinc finger protein 721 (ZNF721), cytochrome P450 family 1 subfamily A member 1 (CYP1A1), cytoplasmic polyadenylation element binding protein 1 (CPEB1), zinc finger protein 275 (ZNF275), mucin 5AC (MUC5AC), serpin family A member 3 (SERPINA3) and DNA topoisomerase II alpha (TOP2A) gene expression. Proteins encoded by these genes are involved in diverse biological pathways, suggesting a broad effect of propylene glycol and glycerol vapours on cellular mechanisms [21].

\section{Inflammatory response and immune cell function}

Air-liquid interface exposure to propylene glycol vapours has been reported to increase the release of pro-inflammatory cytokine interleukin (IL)-6 by lung epithelial cells $[15,16]$. Lung fibroblast exposure to propylene glycol and glycerol exposure does not induce IL-8 production [19]. Exposure to propylene glycol or glycerol does not appear to alter THP-1 macrophages' ability to phagocytose non-typeable Haemophilus influenzae bacteria, with no change in SR-A1 scavenger receptor or Toll-like receptors 2 and 4 (TLR2, TLR4) expression [15]. Culture of phorbol 12-myristate 13-acetate (PMA)-treated human neutrophil cells with propylene glycol and glycerol reduced neutrophil extracellular trap (NET) formation, suggesting alteration of neutrophil antibacterial abilities [22]. Consistent with these results, human blood macrophages and neutrophils exposed to propylene glycol or glycerol showed reduced antimicrobial functions against multi-resistant Streptococcus aeruginosa (MRSA) [18].

In vitro viability tests suggest glycerol and propylene glycol added to the culture media had little to no cytotoxic effects, while exposure to vapours of glycerol and propylene glycol can have cytotoxic effects, possibly through oxidative stress mechanisms. Vapours of propylene glycol and glycerol can also trigger immune response in epithelial cells and/or affect the functionality of immune cells such as neutrophils and macrophages.

\section{Animal studies}

Physiology

Propylene glycol or glycerol exposure does not induce body weight changes in male or female mice [23, 24]. Interestingly, neonatal exposure to propylene glycol and/or glycerol can decrease weight gain in 
offspring $[25,26]$. However, this change in body weight does not translate in a behavioural change in adult mice that were exposed in utero to propylene glycol and glycerol [26].

\section{Lung functions and histology}

As of now, acute exposure to propylene glycol and glycerol does not seem to impact lung functions [27]. More prolonged exposures does alter lung functions, at steady state, following methacholine challenge to assess airway hyperresponsiveness or when combined with tobacco smoke exposure [27-30]. No changes on lung histology in adult mice [28] or mice exposed during the neonatal stage were reported [25]. However, exposure to propylene glycol vapours increase mucociliary clearance [31], as well as the number of MUC5AC positive cells [27]. Propylene glycol and glycerol exposure also modulate the lung lipid mediators, with increased levels of 2-arachidonoylglycerol (2-AG) and 12-hydroxyeicosatetraenoic acid (12-HETE) [30].

\section{Inflammatory response}

Only a few studies investigated the inflammatory response to propylene glycol and glycerol, finding no increase in total bronchoalveolar lavage (BAL) cell numbers in mice following up to 8 weeks of exposure $[29,32,33]$. In the house dust mite (HDM) asthma model, exposure to propylene glycol and glycerol does not appear to affect the immune response, as BAL cell numbers remained unchanged compared to control mice [23]. Another study indicated decreased total BAL cell levels, mainly driven by decreased macrophage numbers [34]. These results concur with other findings indicating no changes in pro-inflammatory cytokine secretion [32] or total BAL protein levels [27] following propylene glycol and glycerol exposure. Propylene glycol and glycerol exposure, alone or with tobacco smoke, also changes lung leukocyte cell frequency [29]. These findings were confirmed in another study, with increased lung dendritic cells, B-cells and CD4+ T-cells [30].

\section{Circadian rhythm}

Propylene glycol and glycerol exposure modulate the expression of key circadian regulatory genes in lung and peripheral organs, alone or combined to tobacco smoke exposure [29, 32, 35]. As circadian rhythm plays a role in a wide array of mechanisms, from immunological to metabolic pathways (reviewed in [36$38]$ ), these findings show that even nicotine-free and flavour-free e-cigarette vapours have the potential to modulate a wide set of pulmonary and systemic biological processes.

Overall, in vivo animal studies suggest that the adverse pulmonary effects of inhaled propylene glycol and glycerol vapours are relatively limited. However, propylene glycol can affect mucociliary clearance and both propylene glycol and glycerol affect the pulmonary expression of genes regulating the circadian rhythm, suggesting these two compounds could change the normal and optimal pulmonary response to pathogens, irritants and allergens.

\section{Clinical studies}

\section{Lung functions}

BOULAY et al. [39] reported that acute propylene glycol and glycerol inhalation $(1 \mathrm{~h})$ do not change lung functions parameters, assessed by spirometry and forced oscillometry, in both healthy and asthmatic subjects, nor does it induce respiratory symptoms. CHAUmont et al. [40] reported reduced transcutaneous oxygen tension in young smokers and subtle changes in forced expiratory volume in $1 \mathrm{~s}\left(\mathrm{FEV}_{1}\right)$ and $\mathrm{FEV}_{1} /$ forced vital capacity ratio.

\section{Inflammatory markers}

A recent study reported no change in BAL immune cells or pro-inflammatory mediators levels in never-smokers and never e-cigarette users following a 2-week use compared to the baseline [41]. Acute propylene glycol and glycerol inhalation $(1 \mathrm{~h})$ does not elevate exhaled nitric oxide fraction or circulating C-reactive protein (CRP) [39]. Acute e-cigarette use causes a slight increase in circulating club-cell secretory protein (CCSP) and surfactant protein (SP)-D levels [40]. Acute propylene glycol and glycerol vaping use does not induce endothelial microparticle formation, a hallmark of vascular damage [42]. Acute propylene glycol and glycerol exposure increases circulating CRP in humans and soluble intercellular adhesion molecule [43].

\section{Gene expression profile}

A 4-week use of propylene glycol and glycerol does not appear to significantly alter mRNA and miRNA levels in bronchial epithelial cells of healthy volunteers [41]. Another gene expression study in acute propylene glycol and glycerol users (20 puffs) reported changes in expression of 65 genes in small airway epithelial cells and 61 genes in alveolar macrophages [42]. Interestingly, this study reported altered expression of key circadian regulatory genes in small airway epithelial cells [42], reinforcing previous preclinical findings [32, 35]. 
Box 1: pulmonary effects of propylene glycol and glycerol

In vitro studies suggest vapours of propylene glycol and glycerol could have cytotoxic effects on lung epithelial cells and other cell types; however, animal and clinical studies do not report signs of extensive lung damage or lung function alteration.

While minor signs of inflammation have been reported in some in vitro, in animal and clinical studies, the general consensus is that exposure to vapours of propylene glycol and glycerol do not trigger a meaningful inflammatory response. However, alterations of the normal function of immune cells have been reported.

While exposure to vapours of propylene glycol and glycerol appears to be well processed by the lungs, more subtle effects such as disruption in lung circadian rhythmicity and mucociliary clearance could impact the response to concomitant exposure to pollutants, allergens and pathogens.

Clinical investigations so far are very limited but support propylene glycol and glycerol having little impact on lung functions, as well as no significant inflammatory effects. There is no doubt inhaling propylene glycol and glycerol is unusual with regard to lung homoeostasis, suggesting effects other than changes in lung functions and inflammation should be observed. Considering alterations in normal lung rhythmicity may reveal insightful for future research. To the best of our knowledge, there are currently no clinical studies assessing the pulmonary impact of chronic propylene glycol and glycerol use, in the absence of nicotine or flavourings.

\section{The attractive: flavours}

Unravelling the effects of vaping flavours on lung biology is by far the most intricate matter in vaping research. As of 2018, over 15000 different flavour blends are commercially available [44]. Investigating the biological impact of flavouring inhalation is a daunting task, as most flavours are often a unique combination of tens, even hundreds, of different molecules. This great variety of flavours is one of the most cited reasons why people choose to initiate e-cigarette use $[45,46]$. While some flavours are relatively straight forward, such as vanilla, strawberry or menthol, some have names that do not evoke any particular flavour, such as "Treasury", "Highlander Grog" or "California Blues" [18]. With that being said, even the simplest flavour names can have a very complex chemical composition [47, 48]. Studying the biological impact of flavouring molecules upon vaporisation is of utmost importance, as pulmonary effects remain largely unknown. In this section, we detail the impact of exposure to e-cigarette liquids or vapours containing flavouring molecules, independently of nicotine.

\section{In vitro studies}

Cellular viability and integrity

Flavour chemicals in e-liquids generate fragmented molecules with oxidative and inflammatory potential $[6,49]$. Some flavours are cytotoxic $[12,50]$. For instance, cinnamaldehyde, responsible for cinnamon flavour, is cytotoxic to endothelial HUVEC/Tert2 cells with increased cell lysis and almost complete cessation of cell metabolic activity [51]. However, other flavours have been shown to only have limited cytotoxic effects [11,52]. Exposure to 2,5-dimethypyrazine, the flavouring compound for chocolate flavour, alters ion conductance in immortalised human bronchial epithelial cells (16HBE14o-), a phenomenon attributed to protein kinase A-dependent activation of the cystic fibrosis transmembrane conductance regulator ion channel [53]. While tobacco-flavoured e-cigarette vapour does not change barrier electrical resistance in primary human small airway epithelial cells [54], cinnamaldehyde exposure decreases ciliary beat frequency in a dose-dependent manner in human bronchial epithelial cells [55].

\section{Inflammatory response}

No change in pro-inflammatory mediator secretion following tobacco- or grape-flavoured e-liquid exposure of human airway epithelial cells (H292) was reported [19]. Other studies showed increased IL-6 production with tobacco-flavoured liquid $[52,54]$. Exposure to cinnamon-flavoured e-liquid increases IL-8 and reactive oxygen species production by human fetal lung fibroblasts [19]. Chemicals such as acetoin and diacetyl (butter flavour), maltol (sweet flavour) and o-vanillin (vanilla flavour) induce IL-8 secretion in bronchial epithelial cells and lung fibroblasts [47].

\section{Immune function}

Tobacco-flavoured e-liquid with or without nicotine increases human rhinovirus load in human epithelial cells, associated with a decreased expression in short palate, lung and nasal epithelial clone-1 (SPLUNC1) receptor [52]. At high concentrations, cinnamaldehyde (cinnamon flavour), ethyl vanillin (vanilla flavour) 
and benzaldehyde (almond or cherry flavour) decrease PMA-stimulated neutrophil capacity to phagocytose Staphylococcus aureus [56], with a decrease in oxygen consumption rate of PMA-stimulated neutrophils. Exposure to isoamyl acetate (banana flavour) had no effect and did not change phagocytosis functions or oxygen consumption rates in neutrophils [56]. Apple-flavoured e-cigarette extract decreases THP-1 macrophages phagocytosis of non-typeable Haemophilus influenzae bacteria, with a reduction in tumour necrosis factor (TNF)- $\alpha$, IL-6, IL-1b, CCL3 and CCL4 secretion, as well as lower surface expression of SR-A1 and TLR2 [15]. Of interest, exposure of Streptococcus pneumoniae to strawberry-flavoured vapour extract changes the expression levels of genes involved in sugar transport and metabolism [57]. Another study reported an increase in pneumococcal penetration of lung epithelial cells following exposure to tobacco-flavoured e-cigarette extract [58].

This shows that several compounds found in e-cigarette vapours derived from flavour molecules can have adverse cytotoxic and inflammatory effects. More studies are needed to assess the specific chemicals that trigger these effects.

\section{Animal studies}

Physiology

While exposure to American tobacco-flavoured e-cigarette vapours does not affect body weight [59], adult mice that were exposed in utero to tobacco-flavoured e-cigarette vapours showed deficits in short-term memory, reduced anxiety and hyperactivity [60].

\section{Lung functions and physiology}

Tobacco-flavoured e-cigarette vapours increase hyperresponsiveness to methacholine, with decreased mucus positive cells in the epithelium compared to air controls [59]. Exposure to vanilla-flavoured vapours increased lung tissue resistance following a methacholine challenge [30]. "Black Liquorice"- and "Kola"-flavoured vapours superimposed to HDM exposure does not change lung reactivity, while "Banana Pudding"-flavoured vapours leads to airway remodelling [23]. Exposure to "Cinnacide"-flavoured vapours increases tissue elastance [23].

\section{Inflammatory response}

Exposure to flavoured e-cigarette vapours (five tested) does not significantly change BAL total cellularity $[23,59]$. Exposure to vanilla-flavoured vapours does not change the number of macrophages within the alveoli [30]. Female mice exposed to tobacco-flavoured e-cigarette vapours showed increased pulmonary IL-1b, IL- 6 and TNF- $\alpha$ levels, and adult offspring of these females also exhibited increased lung TNF- $\alpha$ [61]. Exposure to strawberry-flavoured e-cigarette vapour does not aggravate S. pneumoniae-induced pneumonia in mice [57].

Very few animal studies tackled the pulmonary effects of flavouring agents in vaping and those who did were only able to address a handful of them. Considering these pre-clinical studies act as a proof-of-principle for the potential effects of vaping flavours, it is impossible to deny that these aromatic chemicals can have significant effects on lung homoeostasis.

\section{Clinical studies}

Very few clinical studies use specific e-liquid flavours, or even disclose the flavours used in the study. In fact, only one did and used hazelnut nicotine-free e-liquid to assess acute use of e-cigarette vapours on lung functions. FERRARI et al. [62] indicated acute hazelnut-flavoured e-cigarette use decreased $\mathrm{FEV}_{1}$ and forced expiratory flow at $25 \%$ of forced vital capacity in smokers, with no changes in nonsmokers. However, this study assessed lung functions following 5-min e-cigarette use, which is more representative of a unique and single use than the normal use regiment of e-cigarette users.

Altogether, our current understanding of the pulmonary effects of vaping flavours is negligible. Available data are highly affected by the monstrous number of flavours on the market, making it easier to perform in vitro testing than preclinical and clinical research. However, the pioneers in vaping research definitely established that inhaling flavouring agents can be detrimental to the lungs. We simply do not know which ones and how severe will the consequences.

\section{Box 2: pulmonary effects of vaping flavours}

Great disproportion towards in vitro studies versus preclinical and clinical research

Established potential for flavourings causing cellular cytotoxicity, triggering inflammatory responses, impairing immune function and altering lung functions 
The addictive: nicotine

Nicotine delivery to the lungs was the original purpose when e-cigarettes were brought to market. Lately, reports have shown growing sales of e-liquids containing very high nicotine concentrations $\left(\geqslant 50 \mathrm{mg} \cdot \mathrm{mL}^{-1}\right.$ [63]). Given that the biological effects of nicotine have been studied for decades, this review will be presenting only the new data generated in the context of vaping. In fact, knowing the specific impact of chronic and high-dose nicotine inhalation through e-cigarette vapours is of utmost importance and could potentially diverge from what is already known.

\section{In vitro studies}

Cell viability and gene expression profile

Studies show increased epithelial cell cytotoxicity following nicotine exposure in a dose-dependent manner $[12,13]$. Nicotine-containing vapours also decrease epithelial barrier integrity $[28,64,65]$ and lower ciliary beat frequency in epithelial cells [28]. 24-h exposure of human bronchial epithelial cells to e-cigarette extract with nicotine modulated the expression of 57 genes involved in diverse pathways, such as cell cycle, response to hypoxia, response to organic substance, apoptosis, mitogen-activated protein kinase signalling and acute inflammatory pathways, as well as phospholipid and fatty acid/triacylglycerol metabolism [21]. Solleti et al. [20] also identified 571 miRNAs that were differentially expressed when adding nicotine-containing e-liquid to the culture medium of normal and diseased bronchial epithelial cells.

\section{Immune function}

THP-1 macrophages exposed to nicotine-containing extract showed decreased phagocytic abilities, with decreased secretion of CCL3 and CCL4. They also expressed less SR-A1 and TLR2, suggesting a decreased ability to detect pathogens [15]. The presence of nicotine in e-cigarette extract aggravated the reduction in antimicrobial function against MRSA in mouse alveolar MH-S macrophages and human blood neutrophils [18]. Nicotine vapour extract also affected the virulence of MRSA on its own, with greater biofilm formation and increased adherence and invasion of epithelial cells [18]. Nicotine vapours also directly affect gingival strains of bacteria, with an impact on growth and biofilm formation of oral commensal Streptococcus strains [66].

So far, in vitro studies on nicotine in the context of vaping confirmed several findings that were previously observed, mainly impacts on epithelial cell viability and permeability, as well as its immune-modulatory effects.

\section{Animal studies}

Physiology

Some reports show that exposure to nicotine vapours does not affect weight gain in male [24, 67] or female mice $[23,24]$. Others show weight loss in adult male mice $[23,64,68]$ and in adult males exposed to nicotine vapours at the neonatal stage $[25,26]$. Exposure to nicotine vapours also changes adult mice behaviour, with increased anxiety-like behaviour in male mice, as shown by an increased number of marbles buried when subjected to the marble burring tests [67]. Nicotine vapours also decrease grip strength and swimming abilities in mice [69]. Adult mice exposed in utero to nicotine show behavioural changes as well, with either increased locomotor activity [24] or increased cognitive flexibility when subjected to the water maze test [26].

\section{Lung physiology}

Exposure to nicotine vapour does not cause lung histologic abnormalities in adult mice [18,69]. However, nicotine vapours can affect mucus production in mice, with increased MUC5AC positive cells and expression in lung tissue [28]. Exposure to nicotine vapours during the neonatal stage increases mean linear intercept [25]. The latter observation appears to be caused by a decreased alveolar cell proliferation and lung growth impairment rather than lung damage [25]. Both increase [28] and lack of effect [27] on lung resistance following a methacholine challenge were reported.

\section{Inflammatory response}

Nicotine vapours alone do not generate an influx of immune cells in the mouse lung [18, 23, 27, 28, 33]. Another study reported increased lung tissue neutrophils and $\mathrm{CD}^{+} \mathrm{T}$-lymphocytes in female mice [34]. In a HDM asthma model, exposure to nicotine vapours reduces BAL total cell number, mainly driven by a reduction in eosinophils and macrophages, but increased neutrophils [23]. Mixed reports on inflammatory mediator levels (increase $[18,28]$ or no change [33, 34]) can be found. WANG et al. [34] showed a sex-dependent increase in BAL cytokine levels, with elevated levels in male but not female mice. Nicotine vapours also increase neutrophil myeloperoxidase activity in BAL fluid in mice [34], as well as intracellular phospholipid levels in BAL cells [33]. 
Immune function

Nicotine vapours alter neutrophil functions. In vitro culture of PMA-activated human neutrophil incubated with nicotine, propylene glycol and glycerol decrease NET formation, as well as phagocytosis of S. aureus and Escherichia coli [22]. Nicotine vapours also reduce innate immune response to Pseudomonas aeruginosa, with an increase in peritoneal bacteria burden and decrease in peritoneal neutrophils [22]. Nicotine vapours can also affect pulmonary macrophages. Macrophages harvested from the lungs of mice exposed to nicotine vapours have reduced levels of TNF- $\alpha$, IL-1 $\beta$, NOS2, CD86, CD80 and TLR7 mRNA. Influenza $\mathrm{A}$ infection is more severe in mice exposed to nicotine vapours, with a decrease in survival rates, increase in weight loss, and aggravated lung tissue damage [33].

Nicotine vapours from an e-cigarette have a significant impact on lung development and lung physiology. In vivo animal studies confirm that nicotine is not pro-inflammatory per se, but rather has significant immune-modulatory properties.

\section{Clinical studies}

Very few clinical studies have investigated the specific pulmonary effects of nicotine delivered by an e-cigarette without flavours. A genome-wide gene expression study in small airway epithelial cells and alveolar macrophages from never-smokers following acute e-cigarette use with nicotine found modulation in 71 genes and 27 genes, respectively [42]. Of interest, several genes belonged to the p53 pathway in small airway epithelial cells, but no clear pathways were found in alveolar macrophages [42].

Nicotine found in vaping liquids appears to maintain previously documented properties on lung physiology and the immune system. Nicotine concentrations in vaping liquid can vary greatly; from none to $>50 \mathrm{mg} \cdot \mathrm{mL}^{-1}$. Nicotine poisoning is a well-documented phenomenon in e-cigarette users, and a deeper understanding of the dose-dependent physiological and biological effects on the lung, especially at high doses, may provide novelty.

\section{Box 3: pulmonary effects of nicotine}

Advanced scientific evidence based on decades of research on tobacco smoking

In vitro studies show vapours of nicotine have cytotoxic effects on lung epithelial cells and can impair epithelial barrier integrity. While, animal studies do not report signs of extensive lung damage or lung function alteration, early exposure to nicotine may be detrimental to lung development.

No significant evidence of significant inflammatory effects but well documented immune-modulatory effects and alterations of the normal antimicrobial function of immune cells

\section{Indistinguishable and additive effects of e-cigarette components}

Several studies on vaping do not assess the respective impact of the vehicle, nicotine and flavours (table 1). This leads to very interesting observations that, while being caused by e-cigarettes, cannot be attributed to a specific constituent. The following section details the effects of combined but indistinguishable e-liquid constituents.

\section{In vitro studies}

\section{Cellular viability}

In a study investigating 35 different liquids with different flavours and nicotine concentrations, BAHL et al. [14] showed that e-liquid potency varied greatly depending on cell type used, with greater sensibility using stem cells than pulmonary fibroblasts. There was no correlation between cytotoxicity and nicotine concentration, with the most cytotoxic flavour tested being cinnamon [14]. The degree of cytotoxicity appears to be highly variable within a given flavour group (i.e. fruits, tobacco) [14]. Tobacco-flavoured e-cigarette vapours or extract consistently reduces cell viability [17, 19, 70-73]. LEIGH et al. [74] also found that tobacco, piña colada, menthol, coffee and strawberry flavours decrease cell viability and metabolic activity. Several studies report increased oxidative stress following cell incubation with tobacco-flavoured e-cigarette vapour extract with nicotine [70-73] and with strawberry-flavoured extract with nicotine [75]. Other studies indicate no changes in antioxidant response in epithelial cells exposed to menthol- and tobacco-flavoured e-cigarette vapours $[11,54]$.

\section{Epithelial cell function}

Combined nicotine and flavouring exposure leads to dose-dependent loss of epithelial cell barrier integrity $[76,77]$. Highly concentrated nicotine tobacco- and menthol-flavoured e-cigarette vapours also decreases epithelial cell barrier integrity [78]. Inhibition of Rho kinase and the addition of the sphingosine-1 
TABLE 1 Methodological characteristics of in vitro studies

\section{Electronic cigarette vapour exposure}

Air-liquid interface

E-liquid mixed with cell medium or electronic cigarette vapour extracts

\section{Cell types}

Lung epithelial cells

16HBE (human)

hTBE (human)

A549 cells (human)

NHBE (human)

Beas-2B (human)

H292 (human)

Primary bronchial epithelial cells (human)

Primary small airway epithelial cells (human)

3-dimensional epithelial cell model (human)

Primary tracheal epithelial cells (mouse)

RLEC (rat)

Nasal epithelial cells

HBEpC

3-dimensional epithelial cell model from healthy donors (human)

Immune cells

White blood cells (human)

Blood neutrophils (human)

Blood monocyte-derived dendritic cells (human)

THP-1 monocytic cells (human)

Kupffer cells (rat)

Platelets

Endothelial cells

HUVEC (human)

Primary human microvascular cells-lung derived

MLEC (mouse)

Stem cells

Embryonic stem cells (human)

Bone marrow-derived mesenchymal stem cells (human)

Neural stem cells (mouse)

Lung fibroblasts

Human pulmonary fibroblasts

HFL-1 (human)

Skin cells

HaCaTs (human)

Primary gingival epithelial cells (human)

Periodontal ligament fibroblasts (human)

Lung surfactant

Infasurf (calf)

Bacteria

Streptococcus pneumoniae

Streptococcus gordonii

Streptococcus intermedius

Streptococcus mitis

Streptococcus oralis

\section{Nicotine}

Low nicotine $<6 \mathrm{mg} \cdot \mathrm{mL}^{-1}$

Medium nicotine $6-18 \mathrm{mg} \cdot \mathrm{mL}^{-1}$

High nicotine $>18 \mathrm{mg} \cdot \mathrm{mL}^{-1}$

No nicotine

Unspecified concentration

\section{Flavours}

Tobacco flavours

Menthol

Cinnamon
$[11,12,16,17,19,21,28,34,54,55,65,66,71,73,74,77,78,103]$

$[13-15,18,20,22,47,50-53,56-58,70,72,75,76,79,81,104]$

$[16,53 f, 64]$

[52]

$[12,13,18,50,58,64,73]$

$[17,20,28,65,73]$

$[11,13,19,47,55,58,76]$

$[19,47,74]$

$[16,21,55]$

[54]

$[34,78]$

[53]

[76]

[58]

[77]

[18]

$[22,56,82]$

[104]

[15]

[72]

[81]

$[51,70]$

[76]

[76]

[14]

[75]

[14]

[14]

$[19,47,103]$

$[12,18]$

$[71,80]$

[80]

[79]

[57]

[66]

[66]

[66]

[66]

$[14,19,55,57,70,76]$

$[11,14,15,19,21,47,52,75,77,81,103-105]$

$[13,14,17,19,20,22,28,47,50,54,58,66,71-74,78-80,82,104$, $106]$

$[13,14,16,17,19-21,28,47,53,54,56-58,66,72,73,79-82]$

$[11,14,19,52,54,58,70-74,76-78,80-82,103,105]$

$[11,14,74,78-80]$

[55] 
TABLE 1 Continued

Coffee

Fruit flavours

Dessert and sweet drink flavours

Other

Targeted aromatic molecules

Unflavoured
[47]

$[14,15,19,50,53,57,74,75,79]$

$[14,19,53,74,76]$

[12]

$[19,47,51,53,56]$

$[11,13,14,16,19-22,28,55,66,76,79,104,106]$

HBE: human bronchial epithelial; NHBE: normal and diseased bronchial epithelial.

phosphate receptor 1 (S1P1) agonist restores barrier integrity, suggesting a protective role of S1P1 on epithelial barrier integrity in these conditions [76]. E-liquid induces morphological changes in human lungs and gingival fibroblasts, suggesting a wide range effect of nicotine and/or flavours on several cell types $[19,71]$. Exposure to berry- and menthol-flavoured e-cigarette extract with nicotine do not affect surfactant pressure sustaining proprieties. However, it increases the area between surfactant lipid multilayers, suggesting a potential disruption in surfactant functions [79]. Exposure to tobacco-flavoured e-cigarette vapours with nicotine alters expression of genes involved in metabolic processes, response to organic substances, apoptosis and hypoxia [77].

\section{Inflammatory response}

Tobacco-flavoured liquids with nicotine increase IL-6 production $[52,74]$ and, at high concentrations, decrease MUC5AC production by epithelial cells [77]. Strawberry- and coffee-flavoured vapours with nicotine also induce IL-1 $\beta$, IL-6, IL-10, CXCL1, CXCL2 and CXCL10 secretion [74]. Another study indicates that menthol-flavoured e-cigarette vapours can increase COX2, S100A8, RAGE and $\gamma \mathrm{H} 2 \mathrm{~A} . \mathrm{X}$ protein levels in gingival ligament fibroblasts and gingival epithelium, suggesting a broad range of inflammatory pathway activation across the respiratory tract [80]. Exposure to tobacco-flavoured e-cigarette extract with nicotine increases platelet activation, complement protein expression and deposition, as well as platelet aggregation [72, 81]. Tobacco-flavoured e-cigarette extract containing nicotine increases neutrophil activation in blood neutrophils, with an increase in matrix metalloproteinase-9 levels and CXCL8 secretion, coupled with p38 mitogen-activated protein kinase activation [82].

\section{Immune function}

Tobacco-flavoured e-cigarette extracts with nicotine decrease antimicrobial functions of blood neutrophils against MRSA [18] and increase pneumococcal penetration of lung epithelial cells. Strawberry-flavoured e-cigarette extracts with nicotine change expression levels of genes involved in stress response and metabolism in S. pneumoniae [57], suggesting an impact on pathogens themselves.

\section{Animal studies}

Physiology

Liquorice-, cinnamon- and tobacco-flavoured e-cigarette vapours prevent normal weight gain over time $[23,83]$. Mice exposed to cinnamon-flavoured electronic cigarette vapours containing nicotine before conception and during pregnancy give birth to smaller pups [84]. Adult mice exposed in utero to tobacco-flavoured e-cigarette vapours with nicotine show deficits in short-term memory, reduced anxiety and hyperactivity [60], as well as higher locomotor activity [83].

\section{Lung physiology}

While causing no apparent alterations observable on lung histology [27, 85], tobacco-flavoured e-cigarette exposure containing nicotine increases MUC5AC positive cells [27], as well as lung resistance following a methacholine challenge [27]. Cinnamon-flavoured e-cigarette vapours containing nicotine increase lung elastance in dams [84], with their offspring having increased airspace enlargement at birth; a defect associated with alterations in expression of several genes involved in the Wnt pathway [84].

\section{Inflammatory response}

Animal modelling shows mixed results regarding pulmonary inflammation induced by flavours and nicotine. Some studies report no changes in BAL pro-inflammatory mediator levels [27, 86], while others report higher levels of pro-inflammatory mediator secretion $[19,61]$. Similarly, some studies indicate no 
changes in BAL cellularity [19], while others indicate increased BAL cellularity [27, 86]. With all three studies using whole-body exposures, similar nicotine concentrations and exposure period (table 2), these results can likely be explained by the different e-liquid flavours or types of e-cigarette used.

Immune functions

Mice inoculated with $S$. pneumoniae and exposed for the previous $2 \mathrm{~h}$ to strawberry-flavoured e-cigarette vapour extract containing nicotine do not show any worsening of pneumonia compared to unexposed controls [57]. However, mice exposed to menthol-flavoured e-cigarette vapours with nicotine show decreased abilities to resolve $S$. pneumoniae infection, with more BAL colony forming units (CFU) and less intracellular CFU [86]. Increased nasopharyngeal carriage of $S$. pneumoniae is also found in mice exposed to tobacco-flavoured e-cigarette extract containing nicotine [58]. Mice exposed to menthol-flavoured e-cigarette vapours with nicotine show greater weight loss and mortality following influenza H1N1 infection, with a greater neutrophil recruitment to the airways [86].

\section{Clinical studies}

Several clinical studies assessing e-cigarette health effects had subjects using their own e-cigarette containing both flavoured and nicotine e-liquids, while others provided e-cigarettes for the subjects who most commonly used tobacco-flavoured e-liquids or unflavoured ones (table 3). Short-term e-cigarette use does not change pulmonary function parameters in healthy individuals [87-89]. E-cigarette users have increased sputum and bronchial epithelial MUC5AC levels [90, 91].

\section{TABLE 2 Methodological characteristics of animal studies}

\section{Animals models}

[References]

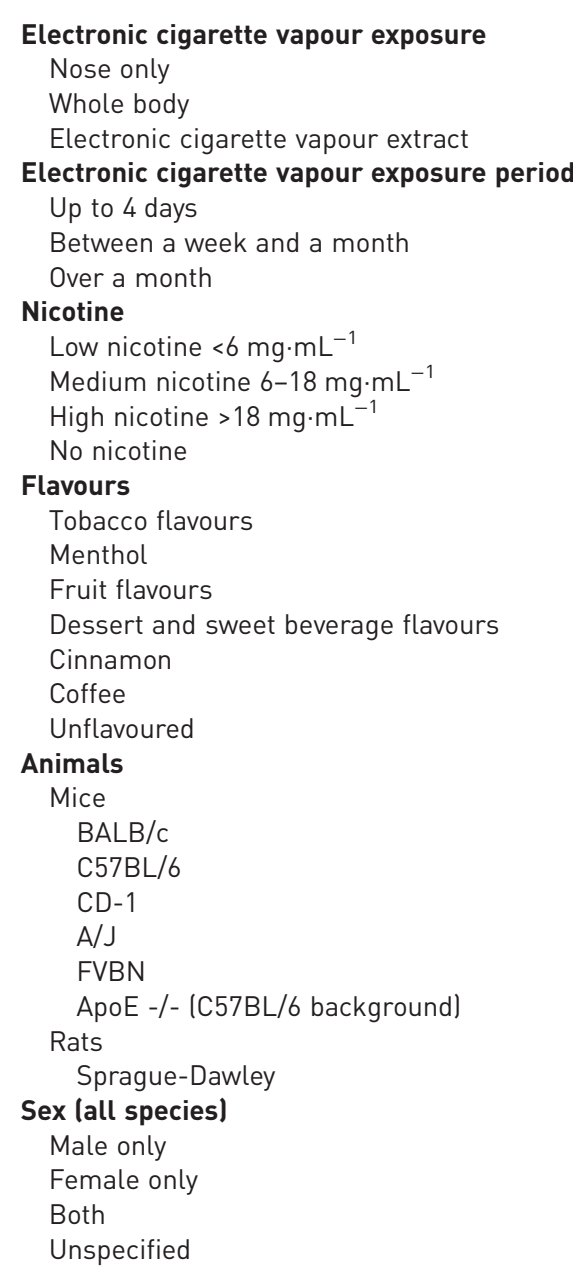

$$
[18,50,64,65]
$$

$[19,23-35,59-61,67-69,83-86,107-109,110]$

[58]

$[19,26,27,34,35,50,58,64]$

$[18,22-25,31,68,69,83,84,86,107,109]$

$[27-30,32,33,59-61,65,67,83,85,108,110]$

$[69,76]$

$[15,18,19,23,24,27,28,50,59,60,67,85,86,107,110]$

$[22,25,26,31,33-35,58,61,65,68,83,84,108,109]$

$[15,23,24,26,27,29,30,32-35,61,69,108]$

$[18,19,27,58,60,61,76,83,107]$

[86]

$[15,18,23,50]$

$[18,23,30,76,108]$

$[23,84]$

$[18,85]$

$[18,22,24-35,59,65,67-69,109,110]$

$[23,29,32,59-61,67,84]$

$[19,22,25-27,30,31,33-35,50,57,65,68,85,86,107,109]$

$[18,24,58,64,65,69]$

[28]

[110]

$[83,108]$

[64]

$[27,31,50,67,83,86,107,110]$

$[18,22,24,25,29,30,32,33,58-60,65,85,108]$

$[23,26,34,35,61,68,84,109]$

$[19,28,57]$ 


\section{TABLE 3 Methodological characteristics of clinical studies}

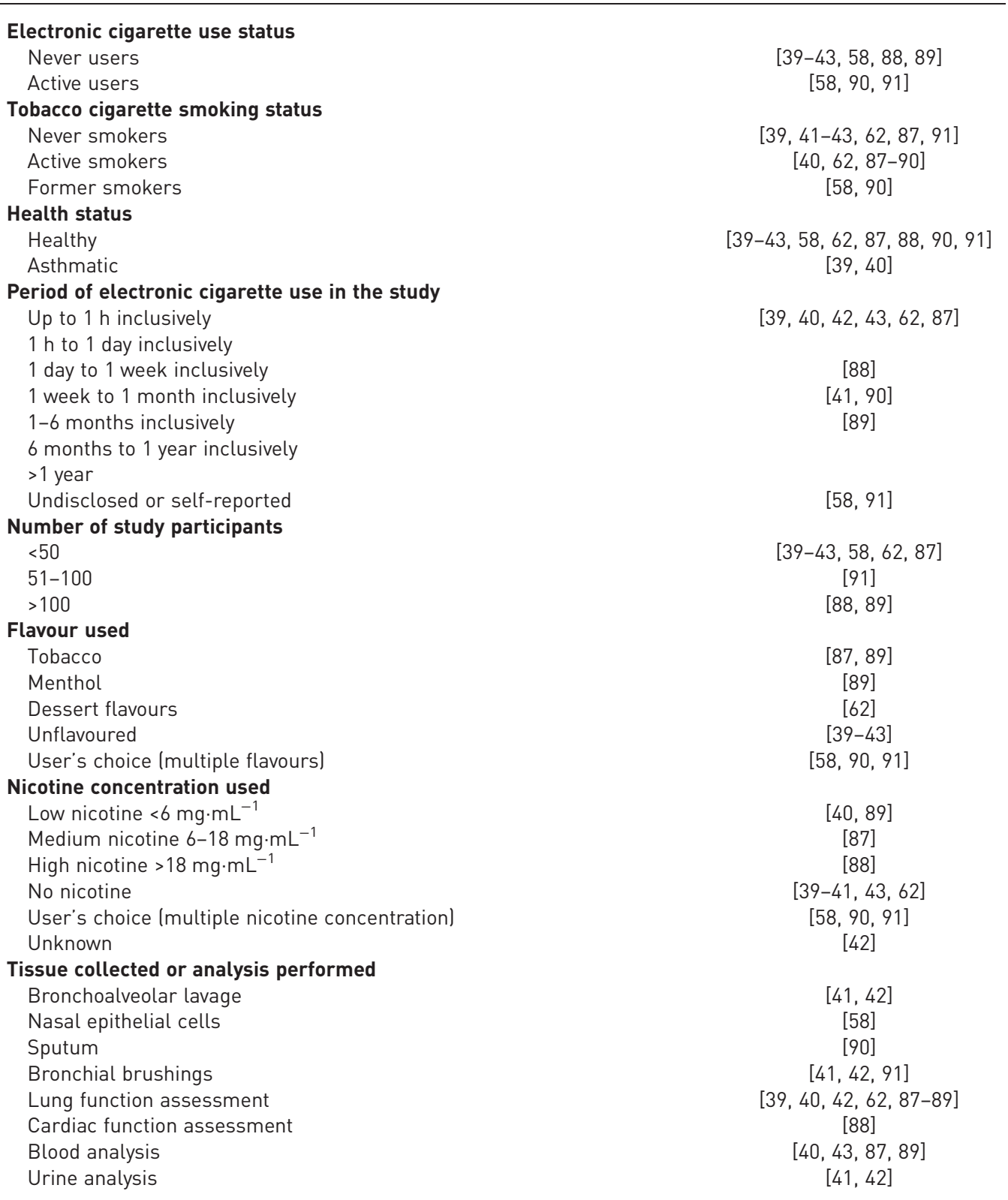

Studies assessing the combined but indistinguishable effects of the propylene glycol, glycerol, nicotine and flavours made more or less the same observations as those who investigated the specific compounds. However, without proper control groups, it remains difficult to assess which e-liquid constituent is responsible for the observed effects.

Box 4: pulmonary effects of vaping that cannot specifically be attributable to a given e-liquid component

Clear detrimental effects of flavoured e-liquids with nicotine on lung epithelial cell viability and cell function in vitro, with most of the studies using tobacco flavours

Adverse effects on airway resistance and lung development

The nature of the inflammatory response triggered by flavoured e-liquids with nicotine is not clear, with mixed results from animal models

Impaired response to bacterial and viral lung infections with worse outcomes 
EVALI: what have we learned from the 2019 epidemic?

In the second half of 2019, a series of reports published in the New England Journal of Medicine reported 78 cases of e-cigarette, or vaping, associated lung injury (EVALI) over the course of just a few mounts [92-95]. These reports raised awareness of the potential harm of acute e-cigarette use, as most cases were young men (mean age 19 years), who mainly vaped for only a few months or years prior to the events. As of February 2020, there have been 2807 hospitalisations associated with EVALI in the USA, of which 50\% of cases reported tetrahydrocannabinol (THC), the main psychoactive constituent of cannabis [96]. In Canada, current data reveal that of the 20 cases of EVALI reported up to April 2020, only five cases reported using THC in their vaping liquids [97]. This highlights the difference between the spike in EVALI in September 2019 in the USA, and the rest of the cases previously and subsequently reported in North America. The presence of vitamin E acetate in illegally sourced e-liquids was been the main culprit in the cases in the USA in 2019 [98]. To date, few in vitro and animal studies have assessed the impact of vitamin $\mathrm{E}$ acetate on pulmonary health. A very brief study exposed mice to vitamin $\mathrm{E}$ acetate for 2 weeks and showed increased BAL albumin levels and CD45+ cells in the lungs, suggesting increased lung inflammation. BHAT et al. [99] also noted the presence of neutral lipid droplets in BAL and lung tissue cells, findings similar to previous case reports [100]. Another study assessed the chemical composition of THC-containing counterfeit e-cigarette products retrieved from EVALI patients. Interestingly, they did not find vitamin $\mathrm{E}$ acetate in all cartridges, but found hydrocarbons, silicates and aldehydes in both liquid and vapour phases of e-cigarette cartridges [101]. Further analysis from this group showed that air-liquid interface exposure of epithelial cells to vapour from counterfeit cartridges induces IL-6 and IL-8, and that vitamin E acetate alone did not have this inflammatory effect [102]. Similar effects were found in mice with increased BAL lipid-laden macrophages, neutrophils and CD4+ T-cells, but not in vitamin E acetate controls [102]. This case is definitely not closed and more studies are needed to find causation and not correlation with e-cigarette liquid constituents as EVALI cases caused by legal vaping liquid use are still being diagnosed in North America.

\section{Thoughts on the future of vaping research}

Our group has been conducting research on vaping for the past 5 years. We were able to identify serious experimental and conceptual limitations and difficulties associated with basic, translational and clinical research on vaping that need to be addressed rapidly to advance our knowledge on the pulmonary and systemic effects of this now not-so-new habit. We humbly make three suggestions to help current and future researchers in their research on vaping.

\section{The need to investigate e-liquid ingredients separately}

This review focuses on highlighting the independent pulmonary effects of each major e-liquid constituent. While many studies used combinations of different e-liquids with and without nicotine and/or flavours, allowing them to pinpoint the constituent responsible for the effects, others did not and used a single or several e-liquids with nicotine and flavour. If we want to identify the constituents with potential detrimental effects to pulmonary health and decipher the underlying mechanisms, we need to know exactly what each constituent can do. This is relatively easy for propylene glycol, glycerol and nicotine, but much more complex for flavours due to their great molecular diversity. In addition, it is much easier to investigate a myriad of different compounds and conditions in in vitro settings than in in vivo animal models or clinical settings. This represents a significant experimental challenge that requires our full attention.

\section{Interactions with other pulmonary conditions and diseases}

E-cigarette users are not only vaping, they smoke, have asthma, are exposed to air pollution, have allergies, catch pneumonia, and have comorbidities (i.e. diabetes, cardiovascular diseases). Pioneering researchers have already started looking at interactions between vaping and other lung diseases and extrapulmonary conditions in animal models. These complex but critical investigations will help enlighten how vaping can impact and interact with other lung irritants and pathogens, as well as comorbidities.

\section{Thinking outside the "smoking" box}

From a chemical and biological point of view, the only similarity between tobacco cigarettes and e-cigarettes is the actual name "cigarette" and the presence of nicotine. Everything else in e-cigarettes has nothing to do with tobacco smoking. So why keep looking for smoking-like effects on the lungs when investigating the pulmonary effects of vaping? Why should vaping cause emphysema or chronic inflammation? Is it possible that vaping may impact lung homeostasis in other ways? Well, it is very likely. Broader omics approaches will likely help unveil unexpected pulmonary effects of vaping. Moreover, the constant comparison between exposure to tobacco cigarette smoke and e-cigarette vapours by assessing biological variable we know are affected by smoking introduce a greater bias in favour of supporting the 
likely false sense of harmlessness of vaping. Meaning that we definitely need to think outside the "smoking" box.

\section{Conclusion}

While there is far more to learn on the effects of e-cigarette use and the underlying mechanisms, it is safe to say that vaping is not innocuous. More properly controlled studies at the cellular, animal and clinical level must be made to assess and regulate the, as yet to be discovered, impact of propylene glycol, glycerol, nicotine and flavours present in e-cigarette liquids.

Conflict of interest: A. Lechasseur has nothing to disclose. M.C. Morissette has nothing to disclose.

\section{References}

1 McMillen RC, Gottlieb MA, Shaefer RM, et al. Trends in electronic cigarette use among U.S. adults: use is increasing in both smokers and nonsmokers. Nicotine Tob Res 2015; 17: 1195-1202.

2 Reid JL, Rynard VL, Czoli CD, et al. Who is using e-cigarettes in Canada? Nationally representative data on the prevalence of e-cigarette use among Canadians. Prev Med 2015; 81: 180-183.

3 Singh T, Arrazola RA, Corey CG, et al. Tobacco use among middle and high school students-United States, 2011-2015. MMWR Morb Mortal Wkly Rep 2016; 65: 361-367.

4 Jensen RP, Strongin RM, Peyton DH. Solvent chemistry in the electronic cigarette reaction vessel. Sci Rep 2017; 7: 42549.

5 Allen JG, Flanigan SS, LeBlanc M, et al. Flavoring chemicals in E-cigarettes: diacetyl, 2,3-pentanedione, and acetoin in a sample of 51 products, including fruit-, candy-, and cocktail-flavored E-cigarettes. Environ Health Perspect 2016; 124: 733-739.

6 Bekki K, Uchiyama S, Ohta K, et al. Carbonyl compounds generated from electronic cigarettes. Int J Environ Res Public Health 2014; 11: 11192-11200.

7 Flora JW, Meruva N, Huang CB, et al. Characterization of potential impurities and degradation products in electronic cigarette formulations and aerosols. Regul Toxicol Pharmacol 2016; 74: 1-11.

8 Jacob SE, Scheman A, McGowan MA. Propylene Glycol. Dermatitis 2018; 29: 3-5.

9 U.S. Food and Drug Administration. CFR: Code of Federal Regulation Title 21. www.accessdata.fda.gov/scripts/ cdrh/cfdocs/cfcfr/CFRSearch.cfm?fr=182.1320 Date last updated: 19 September 2019.

10 Varughese S, Teschke K, Brauer M, et al. Effects of theatrical smokes and fogs on respiratory health in the entertainment industry. Am J Ind Med 2005; 47: 411-418.

11 Antherieu S, Garat A, Beauval N, et al. Comparison of cellular and transcriptomic effects between electronic cigarette vapour and cigarette smoke in human bronchial epithelial cells. Toxicol In Vitro 2017; 45: 417-425.

12 Cervellati F, Muresan XM, Sticozzi C, et al. Comparative effects between electronic and cigarette smoke in human keratinocytes and epithelial lung cells. Toxicol In Vitro 2014; 28: 999-1005.

13 Rankin GD, Wingfors $\mathrm{H}$, Uski O, et al. The toxic potential of a fourth-generation E-cigarette on human lung cell lines and tissue explants. J Appl Toxicol 2019; 39: 1143-1154.

14 Bahl V, Lin S, Xu N, et al. Comparison of electronic cigarette refill fluid cytotoxicity using embryonic and adult models. Reprod Toxicol 2012; 34: 529-537.

15 Ween MP, Whittall JJ, Hamon R, et al. Phagocytosis and Inflammation: exploring the effects of the components of E-cigarette vapour on macrophages. Physiol Rep 2017; 5: e13370.

16 Escobar YH, Nipp G, Cui T, et al. In vitro toxicity and chemical characterization of aerosol derived from electronic cigarette humectants using a newly developed exposure system. Chem Res Toxicol 2020; 33: 1677-1688.

17 Scheffler S, Dieken H, Krischenowski O, et al. Evaluation of E-cigarette liquid vapour and mainstream cigarette smoke after direct exposure of primary human bronchial epithelial cells. Int J Environ Res Public Health 2015; 12: 3915-3925.

18 Hwang JH, Lyes M, Sladewski $\mathrm{K}$, et al. Electronic cigarette inhalation alters innate immunity and airway cytokines while increasing the virulence of colonizing bacteria. J Mol Med 2016; 94: 667-679.

19 Lerner CA, Sundar IK, Yao H, et al. Vapors produced by electronic cigarettes and e-juices with flavorings induce toxicity, oxidative stress, and inflammatory response in lung epithelial cells and in mouse lung. PLoS One 2015; 10: e0116732.

20 Solleti SK, Bhattacharya S, Ahmad A, et al. MicroRNA expression profiling defines the impact of electronic cigarettes on human airway epithelial cells. Sci Rep 2017; 7: 1081.

21 Shen Y, Wolkowicz MJ, Kotova $\mathrm{T}$, et al. Transcriptome sequencing reveals e-cigarette vapour and mainstream-smoke from tobacco cigarettes activate different gene expression profiles in human bronchial epithelial cells. Sci Rep 2016; 6: 23984.

22 Corriden R, Moshensky A, Bojanowski CM, et al. E-cigarette use increases susceptibility to bacterial infection by impairment of human neutrophil chemotaxis, phagocytosis, and NET formation. Am J Physiol Cell Physiol 2020; 318: C205-C214.

23 Chapman DG, Casey DT, Ather JL, et al. The effect of flavored E-cigarettes on murine allergic airways disease. Sci Rep 2019; 9: 13671.

24 Church JS, Chace-Donahue F, Blum JL, et al. Neuroinflammatory and behavioral outcomes measured in adult offspring of mice exposed prenatally to E-cigarette aerosols. Environ Health Perspect 2020; 128: 47006.

25 McGrath-Morrow SA, Hayashi M, Aherrera A, et al. The effects of electronic cigarette emissions on systemic cotinine levels, weight and postnatal lung growth in neonatal mice. PLoS One 2015; 10: e0118344.

26 Smith D, Aherrera A, Lopez A, et al. Adult behavior in male mice exposed to E-cigarette nicotine vapors during late prenatal and early postnatal life. PLoS One 2015; 10: e0137953.

27 Glynos C, Bibli SI, Katsaounou P, et al. Comparison of the effects of e-cigarette vapor with cigarette smoke on lung function and inflammation in mice. Am J Physiol Lung Cell Mol Physiol 2018; 315: L662-L672. 
Garcia-Arcos I, Geraghty P, Baumlin N, et al. Chronic electronic cigarette exposure in mice induces features of COPD in a nicotine-dependent manner. Thorax 2016; 71: 1119-1129.

Lechasseur A, Huppe CA, Talbot M, et al. Exposure to nicotine-free and flavor-free e-cigarette vapors modifies the pulmonary response to tobacco cigarette smoke in female mice. Am J Physiol Lung Cell Mol Physiol 2020; in press [https://doi.org/10.1152/ajplung.00037.2020].

Szafran BN, Pinkston R, Perveen Z, et al. Electronic-cigarette vehicles and flavoring affect lung function and immune responses in a murine model. Int J Mol Sci 2020; 21: 6022.

Laube BL, Afshar-Mohajer N, Koehler K, et al. Acute and chronic in vivo effects of exposure to nicotine and propylene glycol from an E-cigarette on mucociliary clearance in a murine model. Inhal Toxicol 2017; 29: 197-205.

Lechasseur A, Jubinville E, Routhier J, et al. Exposure to electronic cigarette vapors affects pulmonary and systemic expression of circadian molecular clock genes. Physiol Rep 2017; 5: e13440.

Madison MC, Landers $\mathrm{CT}, \mathrm{Gu} \mathrm{BH}$, et al. Electronic cigarettes disrupt lung lipid homeostasis and innate immunity independent of nicotine. J Clin Invest 2019; 129: 4290-4304.

Wang Q, Khan NA, Muthumalage $\mathrm{T}$, et al. Dysregulated repair and inflammatory responses by e-cigarette-derived inhaled nicotine and humectant propylene glycol in a sex-dependent manner in mouse lung. FASEB Bioadv 2019; 1: 609-623.

Khan NA, Yogeswaran S, Wang Q, et al. Waterpipe smoke and e-cigarette vapour differentially affect circadian molecular clock gene expression in mouse lungs. PLoS One 2019; 14: e0211645.

Dibner C, Schibler U, Albrecht U. The mammalian circadian timing system: organization and coordination of central and peripheral clocks. Annu Rev Physiol 2010; 72: 517-549.

Dickmeis T. Glucocorticoids and the circadian clock. J Endocrinol 2009; 200: 3-22.

Scheiermann C, Kunisaki Y, Frenette PS. Circadian control of the immune system. Nat Rev Immunol 2013; 13: 190-198.

Boulay ME, Henry C, Bosse Y, et al. Acute effects of nicotine-free and flavour-free electronic cigarette use on lung functions in healthy and asthmatic individuals. Respir Res 2017; 18: 33.

Chaumont $\mathrm{M}$, van de Borne $\mathrm{P}$, Bernard $\mathrm{A}$, et al. Fourth generation e-cigarette vaping induces transient lung inflammation and gas exchange disturbances: results from two randomized clinical trials. Am J Physiol Lung Cell Mol Physiol 2019; 316: L705-L719.

Song MA, Reisinger SA, Freudenheim JL, et al. Effects of electronic cigarette constituents on the human lung: a pilot clinical trial. Cancer Prev Res (Phila) 2020; 13: 145-152.

Staudt MR, Salit J, Kaner RJ, et al. Altered lung biology of healthy never smokers following acute inhalation of E-cigarettes. Respir Res 2018; 19: 78

Chatterjee S, Tao JQ, Johncola A, et al. Acute exposure to e-cigarettes causes inflammation and pulmonary endothelial oxidative stress in nonsmoking, healthy young subjects. Am J Physiol Lung Cell Mol Physiol 2019; 317: L155-L166. brand websites. J Med Internet Res 2018; 20: e80.

Symes YR, Ribisl KM, Boynton MH, et al. Dual cigarette and e-cigarette use in cancer survivors: an analysis using Population Assessment of Tobacco Health (PATH) data. J Cancer Surviv 2019; 13: 161-170.

Smets J, Baeyens F, Chaumont M, et al. When less is more: vaping low-nicotine vs. high-nicotine e-liquid is compensated by increased wattage and higher liquid consumption. Int J Environ Res Public Health 2019; 16: 723.

Gerloff J, Sundar IK, Freter R, et al. Inflammatory response and barrier dysfunction by different E-cigarette flavoring chemicals identified by gas chromatography-mass spectrometry in E-liquids and E-vapors on human lung epithelial cells and fibroblasts. Appl In Vitro Toxicol 2017; 3: 28-40.

Tierney PA, Karpinski CD, Brown JE, et al. Flavour chemicals in electronic cigarette fluids. Tob Control 2016; 25: e10-e15.

Kopa PN, Pawliczak R. Effect of smoking on gene expression profile - overall mechanism, impact on respiratory system function, and reference to electronic cigarettes. Toxicol Mech Methods 2018; 28: 397-409.

Husari A, Shihadeh A, Talih S, et al. Acute exposure to electronic and combustible cigarette aerosols: effects in an animal model and in human alveolar cells. Nicotine Tob Res 2016; 18: 613-619.

Noel JC, Rainer D, Gstir R, et al. Quantification of selected aroma compounds in e-cigarette products and toxicity evaluation in HUVEC/Tert2 cells. Biomed Chromatogr 2020; 34: e4761.

Wu $\mathrm{Q}$, Jiang $\mathrm{D}$, Minor $\mathrm{M}$, et al. Electronic cigarette liquid increases inflammation and virus infection in primary human airway epithelial cells. PLoS One 2014; 9: e108342.

Sherwood CL, Boitano S. Airway epithelial cell exposure to distinct e-cigarette liquid flavorings reveals toxicity thresholds and activation of CFTR by the chocolate flavoring 2,5-dimethypyrazine. Respir Res 2016; 17: 57. Gellatly S, Pavelka N, Crue T, et al. Nicotine-free E-cigarette vapor exposure stimulates IL6 and mucin production in human primary small airway epithelial cells. J Inflamm Res 2020; 13: 175-185.

Clapp PW, Lavrich KS, van Heusden CA, et al. Cinnamaldehyde in flavored e-cigarette liquids temporarily suppresses bronchial epithelial cell ciliary motility by dysregulation of mitochondrial function. Am J Physiol Lung Cell Mol Physiol 2019; 316: L470-L486.

Hickman E, Herrera CA, Jaspers I. Common E-cigarette flavoring chemicals impair neutrophil phagocytosis and oxidative burst. Chem Res Toxicol 2019; 32: 982-985.

Bagale K, Paudel S, Cagle H, et al. Electronic cigarette (E-cigarette) vapor exposure alters the Streptococcus pneumoniae transcriptome in a nicotine-dependent manner without affecting pneumococcal virulence. Appl Environ Microbiol 2020; 86: e02125-19.

Miyashita L, Suri R, Dearing E, et al. E-cigarette vapour enhances pneumococcal adherence to airway epithelial cells. Eur Respir J 2018; 51: 1701592.

Larcombe AN, Janka MA, Mullins BJ, et al. The effects of electronic cigarette aerosol exposure on inflammation and lung function in mice. Am J Physiol Lung Cell Mol Physiol 2017; 313: L67-L79.

Nguyen T, Li GE, Chen $\mathrm{H}$, et al. Maternal E-cigarette exposure results in cognitive and epigenetic alterations in offspring in a mouse model. Chem Res Toxicol 2018; 31: 601-611. 
Chen H, Li G, Chan YL, et al. Maternal E-cigarette exposure in mice alters DNA methylation and lung cytokine expression in offspring. Am J Respir Cell Mol Biol 2018; 58: 366-377.

Ferrari M, Zanasi A, Nardi E, et al. Short-term effects of a nicotine-free e-cigarette compared to a traditional cigarette in smokers and non-smokers. BMC Pulm Med 2015; 15: 120.

Romberg AR, Miller Lo EJ, Cuccia AF, et al. Patterns of nicotine concentrations in electronic cigarettes sold in the United States, 2013-2018. Drug Alcohol Depend 2019; 203: 1-7.

Ahmad S, Zafar I, Mariappan N, et al. Acute pulmonary effects of aerosolized nicotine. Am J Physiol Lung Cell Mol Physiol 2019; 316: L94-L104.

Crotty Alexander LE, Drummond CA, Hepokoski M, et al. Chronic inhalation of E-cigarette vapor containing nicotine disrupts airway barrier function and induces systemic inflammation and multi-organ fibrosis in mice. Am J Physiol Regul Integr Comp Physiol 2018; 314: R834-R847.

Cuadra GA, Smith MT, Nelson JM, et al. A Comparison of flavorless electronic cigarette-generated aerosol and conventional cigarette smoke on the survival and growth of common oral commensal Streptococci. Int J Environ Res Public Health 2019; 16: 1669.

Ponzoni L, Moretti M, Sala M, et al. Different physiological and behavioural effects of e-cigarette vapour and cigarette smoke in mice. Eur Neuropsychopharmacol 2015; 25: 1775-1786.

Shi H, Fan X, Horton A, et al. The effect of electronic-cigarette vaping on cardiac function and angiogenesis in mice. Sci Rep 2019; 9: 4085.

Chen YM, Huang CC, Sung HC, et al. Electronic cigarette exposure reduces exercise performance and changes the biochemical profile of female mice. Biosci Biotechnol Biochem 2019; 83: 2318-2326.

Anderson C, Majeste A, Hanus J, et al. E-cigarette aerosol exposure induces reactive oxygen species, DNA damage, and cell death in vascular endothelial cells. Toxicol Sci 2016; 154: 332-340.

Rouabhia M, Park HJ, Semlali A, et al. E-cigarette vapor induces an apoptotic response in human gingival epithelial cells through the caspase-3 pathway. J Cell Physiol 2017; 232: 1539-1547.

Rubenstein DA, Hom S, Ghebrehiwet B, et al. Tobacco and e-cigarette products initiate Kupffer cell inflammatory responses. Mol Immunol 2015; 67: 652-660.

Scheffler S, Dieken H, Krischenowski $\mathrm{O}$, et al. Cytotoxic evaluation of E-liquid aerosol using different lung-derived cell models. Int J Environ Res Public Health 2015; 12: 12466-12474.

Leigh NJ, Lawton RI, Hershberger PA, et al. Flavourings significantly affect inhalation toxicity of aerosol generated from electronic nicotine delivery systems (ENDS). Tob Control 2016; 25: Suppl. 2, ii81-ii87.

Shaito A, Saliba J, Husari A, et al. Electronic cigarette smoke impairs normal mesenchymal stem cell differentiation. Sci Rep 2017; 7: 14281.

Schweitzer KS, Chen SX, Law S, et al. Endothelial disruptive proinflammatory effects of nicotine and e-cigarette vapour exposures. Am J Physiol Lung Cell Mol Physiol 2015; 309: L175-L187.

Haswell LE, Baxter A, Banerjee A, et al. Reduced biological effect of e-cigarette aerosol compared to cigarette smoke evaluated in vitro using normalized nicotine dose and RNA-seq-based toxicogenomics. Sci Rep 2017; 7: 888.

Neilson L, Mankus C, Thorne D, et al. Development of an in vitro cytotoxicity model for aerosol exposure using 3D reconstructed human airway tissue; application for assessment of e-cigarette aerosol. Toxicol In Vitro 2015; 29: $1952-1962$.

Przybyla RJ, Wright J, Parthiban R, et al. Electronic cigarette vapour alters the lateral structure but not tensiometric properties of calf lung surfactant. Respir Res 2017; 18: 193.

Sundar IK, Javed F, Romanos GE, et al. E-cigarettes and flavorings induce inflammatory and pro-senescence responses in oral epithelial cells and periodontal fibroblasts. Oncotarget 2016; 7: 77196-77204.

Hom S, Chen L, Wang T, et al. Platelet activation, adhesion, inflammation, and aggregation potential are altered in the presence of electronic cigarette extracts of variable nicotine concentrations. Platelets 2016; 27: 694-702.

Higham A, Rattray NJ, Dewhurst JA, et al. Electronic cigarette exposure triggers neutrophil inflammatory responses. Respir Res 2016; 17: 56.

Shao XM, Lopez B, Nathan D, et al. A mouse model for chronic intermittent electronic cigarette exposure exhibits nicotine pharmacokinetics resembling human vapers. J Neurosci Methods 2019; 326: 108376.

Noel A, Hansen S, Zaman A, et al. In utero exposures to electronic-cigarette aerosols impair the Wnt signaling during mouse lung development. Am J Physiol Lung Cell Mol Physiol 2020; 318: L705-L722.

Olfert IM, DeVallance E, Hoskinson $\mathrm{H}$, et al. Chronic exposure to electronic cigarettes results in impaired cardiovascular function in mice. J Appl Physiol 2018; 124: 573-582.

Sussan TE, Gajghate S, Thimmulappa RK, et al. Exposure to electronic cigarettes impairs pulmonary anti-bacterial and anti-viral defenses in a mouse model. PLoS One 2015; 10: e0116861.

Flouris AD, Chorti MS, Poulianiti KP, et al. Acute impact of active
serum cotinine and lung function. Inhal Toxicol 2013; 25: 91-101.

D'Ruiz CD, O'Connell G, Graff DW, et al. Measurement of cardiovascular and pulmonary function endpoints and other physiological effects following partial or complete substitution of cigarettes with electronic cigarettes in adult smokers. Regul Toxicol Pharmacol 2017; 87: 36-53.

Cravo AS, Bush J, Sharma G, et al. A randomised, parallel group study to evaluate the safety profile of an electronic vapour product over 12 weeks. Regul Toxicol Pharmacol 2016; 81: Suppl. 1, S1-S14.

Reidel B, Radicioni G, Clapp PW, et al. E-cigarette use causes a unique innate immune response in the lung involving increased neutrophilic activation and altered mucin secretion. Am J Respir Crit Care Med 2018; 197: 492-501.

1 Ghosh A, Coakley RC, Mascenik T, et al. Chronic E-cigarette exposure alters the human bronchial epithelial proteome. Am J Respir Crit Care Med 2018; 198: 67-76.

Henry TS, Kanne JP, Kligerman SJ. Imaging of vaping-associated lung disease. $N$ Engl J Med 2019; 381: 1486-1487.

Layden JE, Ghinai I, Pray I, et al. Pulmonary illness related to E-cigarette use in Illinois and Wisconsin - final report. N Engl J Med 2020; 382: 903-916.

Maddock SD, Cirulis MM, Callahan SJ, et al. Pulmonary lipid-laden macrophages and vaping. N Engl J Med 2019; 381: 1488-1489. 
Christiani DC. Vaping-induced acute lung injury. N Engl J Med 2020; 382: 960-962.

Centers for Disease Control and Prevention. Outbreak of Lung Injury Associated with E-Cigarette use, or Vaping. cdc.gov/tobacco/basic_information/e-cigarettes/severe-lung-disease.html\#latest-information Date last updated: 25 February 2020; date last accessed: 11 August 2020.

Government of Canada. Vaping-associated lung illness. www.canada.ca/en/public-health/services/diseases/vapingpulmonary-illness.html Date last updated: 20 August 2020; date last accessed: 11 August 2020.

Blount BC, Karwowski MP, Shields PG, et al. Vitamin E acetate in bronchoalveolar-lavage fluid associated with EVALI. N Engl J Med 2020; 382: 697-705.

Bhat TA, Kalathil SG, Bogner PN, et al. An animal model of inhaled vitamin E acetate and EVALI-like lung injury. N Engl J Med 2020; 382: 1175-1177.

Parraga G, Morissette MC. E-cigarettes: what evidence links vaping to acute lung injury and respiratory failure? Can J Respir Crit Care Sleep Med 2020; 4: 48-54.

Muthumalage T, Friedman MR, McGraw MD, et al. Chemical constituents involved in E-Cigarette, or vaping product use-associated lung injury (EVALI). Toxics 2020; 8: 25.

Muthumalage T, Lucas JH, Wang Q, et al. Pulmonary toxicity and inflammatory response of E-cigarette vape cartridges containing medium-chain triglycerides oil and vitamin E acetate: implications in the pathogenesis of EVALI. Toxics 2020; 8: E46.

3 Lerner CA, Rutagarama P, Ahmad T, et al. Electronic cigarette aerosols and copper nanoparticles induce mitochondrial stress and promote DNA fragmentation in lung fibroblasts. Biochem Biophys Res Commun 2016; 477: 620-625.

Chen IL, Todd I, Tighe PJ, et al. Electronic cigarette vapour moderately stimulates pro-inflammatory signalling pathways and interleukin-6 production by human monocyte-derived dendritic cells. Arch Toxicol 2020; 94: 2097-2112.

Breheny $\mathrm{D}$, Oke $\mathrm{O}$, Pant $\mathrm{K}$, et al. Comparative tumor promotion assessment of e-cigarette and cigarettes using the in vitro Bhas 42 cell transformation assay. Environ Mol Mutagen 2017; 58: 190-198.

Crotty Alexander LE, Drummond CA, Hepokoski M, et al. Chronic inhalation of e-cigarette vapour containing nicotine disrupts airway barrier function and induces systemic inflammation and multiorgan fibrosis in mice. Am J Physiol Regul Integr Comp Physiol 2018; 314: R834-R847.

07 Zelikoff JT, Parmalee NL, Corbett K, et al. Microglia activation and gene expression alteration of neurotrophins in the hippocampus following early-life exposure to E-cigarette aerosols in a murine model. Toxicol Sci 2018; 162: $276-286$

Szostak J, Wong ET, Titz B, et al. A 6-month systems toxicology inhalation study in ApoE(-/-) mice demonstrates reduced cardiovascular effects of E-vapour aerosols compared with cigarette smoke. Am J Physiol Heart Circ Physiol 2020; 318: H604-H631.

Wetendorf M, Randall LT, Lemma MT, et al. E-cigarette exposure delays implantation and causes reduced weight gain in female offspring exposed in utero. J Endocr Soc 2019; 3: 1907-1916.

Lee HW, Park SH, Weng MW, et al. E-cigarette smoke damages DNA and reduces repair activity in mouse lung, heart, and bladder as well as in human lung and bladder cells. Proc Natl Acad Sci USA 2018; 115: E1560-E1569. 